\title{
Observation of Elastic Doublon Decay in the Fermi-Hubbard Model
}

\author{
Niels Strohmaier, Daniel Greif, Robert Jördens, Leticia Tarruell, Henning Moritz ${ }^{*}$ and Tilman Esslinger \\ Institute for Quantum Electronics, ETH Zurich, 8093 Zurich, Switzerland
}

\author{
Rajdeep Sensarma, ${ }^{1,2}$ David Pekker, ${ }^{1}$ Ehud Altman, ${ }^{3}$ and Eugene Demler ${ }^{1}$ \\ ${ }^{1}$ Department of Physics, Harvard University, Cambridge, Massachusetts 02138, USA \\ ${ }^{2}$ Condensed Matter Theory Center, University of Maryland, College Park, Maryland 20742, USA and \\ ${ }^{3}$ Department of Condensed Matter Physics, Weizmann Institute, Rehovot, 76100, Israel
}

(Dated: October 25, 2018)

\begin{abstract}
We investigate the decay of highly excited states of ultracold fermions in a three-dimensional optical lattice. Starting from a repulsive Fermi-Hubbard system near half filling, we generate additional doubly occupied sites (doublons) by lattice modulation. The subsequent relaxation back to thermal equilibrium is monitored over time. The measured absolute doublon lifetime covers two orders of magnitude. In units of the tunneling time $h / J$ it is found to depend exponentially on the ratio of on-site interaction energy $U$ to kinetic energy $J$. We argue that the dominant mechanism for the relaxation is a simultaneous many-body process involving several single fermions as scattering partners. A many-body calculation is carried out using diagrammatic methods, yielding fair agreement with the data.
\end{abstract}

PACS numbers: 05.30.Fk, 03.75.Ss, 67.85.-d, 71.10.Fd

Understanding the far-from-equilibrium dynamics of strongly correlated systems is a highly challenging task. Even the identification of the basic processes involved and the associated time scales is nontrivial when the system cannot be described by weakly interacting excitations or quasiparticles. In these systems, dynamics may couple states with widely different energies making the description in terms of a restricted set of low energy states impossible. While progress has been achieved for one-dimensional systems (11, 2], and references therein), these results can typically not be extended to higher dimensions.

The main difficulty in analyzing non-equilibrium dynamics in the setting of condensed matter experiments is the strong coupling to the environment, which introduces extrinsic relaxation mechanisms and makes it challenging to prepare far-from-equilibrium initial states in a controlled way. By contrast, the nearly perfect isolation of many-body systems realized with ultracold atoms makes them a perfect candidate for studying the intrinsic dynamics of strongly correlated systems. In the setting of ultracold atoms it is possible to prepare a well-controlled initial state, evolve it under the action of a precisely defined microscopic Hamiltonian, and monitor the effects of the characteristic relaxation process 3 .

In this Letter, we take advantage of the recent realization of the repulsive Fermi-Hubbard model with ultracold atom systems [4 6] to investigate the relaxation of artificially created highly excited states. This problem appears in diverse contexts like multiphonon decay of excitons in semiconductors [7, pump-probe experiments [8] and dynamics of resonances in nuclear matter [9. Due to the negligible coupling to an external environment, we are able to carry out a direct comparison of experiment and theory. The interpretation of these results shows the importance of high-order scattering processes in bridging the energy gap between low- and high-energy excitations and how they can lead to exponentially slow thermalization.

In the experiment, we study the time evolution of doubly occupied lattice sites (doublons) in the repulsive Fermi-Hubbard model. This model describes fermionic particles hopping on a lattice with tunneling $J$ and onsite repulsion $U$ and is realized by a two-component Fermi gas in an optical lattice. In the context of a dilute Bose-Hubbard system isolated repulsively bound pairs have been experimentally identified and studied [10].

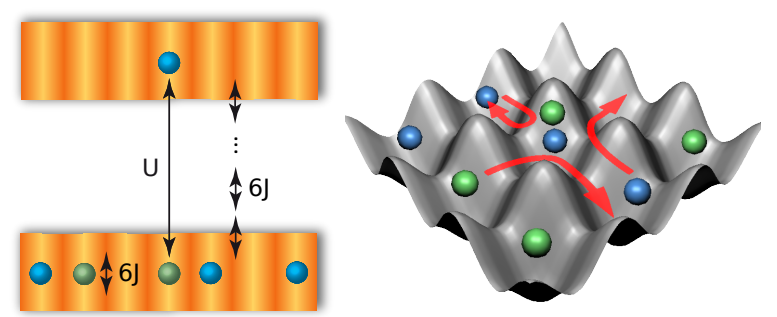

FIG. 1: Stability of highly excited states in the single-band Hubbard model. Doubly occupied lattice sites are protected against decay by the on-site interaction energy $U$. The average kinetic energy of a single particle in a periodic potential is half the bandwidth $6 J$. Thus the relaxation of excitations requires several scattering partners to maintain energy conservation.

We report on the observation of elastic decay of artificially created doublons 5, 11, 12 into single particles. The resulting lifetime is found to increase exponentially with the ratio $U / 6 J$ (i.e. the lifetime becomes longer as the interactions become stronger). We argue that a doublon, having an excess energy $U$, decays in a scatter- 
ing process involving several single fermions, cf. Fig. 1. Since each of these scattering partners can only absorb an average energy of $6 J$, the number of virtual states involved in the simultaneous many-body process is $U / 6 \mathrm{~J}$. Hence the decay is exponentially suppressed for increasing $U / 6 J$. We find fair agreement with diagrammatic calculations where the strongly correlated nature of the underlying state is crucial in obtaining the correct value of the scaling exponent.

The experimental sequence used to produce quantum degenerate Fermi gases has been described in detail in Ref. [5]. In brief, we prepare $(50 \pm 5) \times 10^{3}{ }^{40} \mathrm{~K}$ atoms at temperatures below $15 \%$ of the Fermi temperature $T_{\mathrm{F}}$ in a balanced mixture of two magnetic sublevels of the $F=9 / 2$ manifold. The confinement is given by a dipole trap with trapping frequencies $\omega_{x, y, z}=$ $2 \pi \times(35,23,120) \mathrm{Hz}$. Using Feshbach resonances in either a $\left(m_{\mathrm{F}}=-9 / 2,-7 / 2\right)$ or $\left(m_{\mathrm{F}}=-9 / 2,-5 / 2\right)$ mixture [13, 14], the interaction strength is tuned in the range $98 a_{0}-131 a_{0}$ or $374 a_{0}-672 a_{0}$ respectively, where $a_{0}$ is the Bohr radius. After adjusting the scattering length to the desired value, we add a three-dimensional cubic optical lattice. The lattice depth is increased in $200 \mathrm{~ms}$ to final values between $6.5 E_{\mathrm{R}}$ and $12.5 E_{\mathrm{R}}$ in units of the recoil energy $E_{\mathrm{R}}=h^{2} / 2 m \lambda^{2}$. Here $\lambda=1064 \mathrm{~nm}$ is the wavelength of the lattice beams. The lattice beams have Gaussian profiles with $1 / e^{2}$ radii of $w_{x, y, z}=(160,180,160) \mu \mathrm{m}$. For a given scattering length and lattice depth, $J$ and $U$ are inferred from Wannier functions [16]. Their statistical and systematic errors are dominated by the lattice calibration and the accuracies in width and position of the two Feshbach resonances 13, 14. Depending on $U$ and $J$ the accessible final regimes of the system range from metallic to Mott insulating phases with a double occupancy below $15 \%$.

The preparation of the system is followed by a sinusoidal modulation of the lattice depth with an amplitude of $10 \%$ and frequency close to $U / h$. This causes an increase of the double occupancy to values up to $35 \%$ [17, 18.

After the modulation the system is in a nonequilibrium state, which we let evolve freely at the initial lattice depth and interaction strength for up to $4 \mathrm{~s}$. This is followed by a sudden increase of the lattice depth to $30 E_{\mathrm{R}}$, which prevents further tunneling. We then measure the amount of atoms residing on singly (doubly) occupied sites $N_{\mathrm{s}}\left(N_{\mathrm{d}}\right)$ by encoding the double occupancy into a previously unpopulated spin state using RF spectroscopy 5. Combining Stern-Gerlach separation and absorption imaging we obtain the single occupancy $n_{\mathrm{s}}=N_{\mathrm{s}} / N_{\text {tot }}$, double occupancy $n_{\mathrm{d}}=N_{\mathrm{d}} / N_{\text {tot }}$ and total atom number $N_{\text {tot }}=N_{\mathrm{s}}+N_{\mathrm{d}}$.

We record the time evolution of the total atom number and the single and double occupancy, see Fig. 2. The double occupancy is found to decay exponentially, while additional losses are also observed on longer timescales, which lead to a reduction of the total atom number. To extract the doublon lifetime we model these decays by a set of coupled rate equations:

$$
\begin{aligned}
\Delta \dot{N}_{\mathrm{d}} & =-\left(\frac{1}{\tau_{\mathrm{D}}}+\frac{1}{\tau_{\text {in }}}+\frac{1}{\tau_{\text {loss }}}\right) \Delta N_{\mathrm{d}} \\
\dot{N}_{\mathrm{d}, 0} & =-\left(\frac{1}{\tau_{\text {in }}}+\frac{1}{\tau_{\text {loss }}}\right) N_{\mathrm{d}, 0} \\
\dot{N}_{\mathrm{s}} & =\frac{1}{\tau_{\mathrm{D}}} \Delta N_{\mathrm{d}}-\frac{1}{\tau_{\text {loss }}} N_{\mathrm{s}}
\end{aligned}
$$

Here $\Delta N_{\mathrm{d}}$ is the additional amount of double occupancy created by the lattice modulation as compared to the equilibrium population $N_{\mathrm{d}, 0}$. The three time constants correspond to three independent local decay processes differing in the type of site they affect: the lifetime of doublons $\tau_{\mathrm{D}}$ describes a population flow from doubly occupied to singly occupied lattice sites visible as a fast decay (rise) of double (single) occupancy within $0.01-1 \mathrm{~s}$. The other two times denote loss time constants, which lead to a reduction of the total atom number: $\tau_{\text {loss }}$ corresponds to losses affecting both site types in the same manner, which is only observed in the total atom number. Additional inelastic losses on doubly occupied sites are summarized by $\tau_{\text {in }}$, visible as a simultaneous decay of both the total atom number and double occupancy. Changes of the decay times during the decay and higher order terms in the rate equations are excluded.
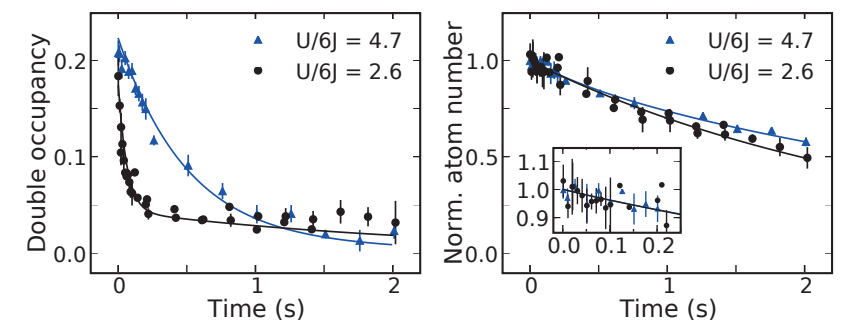

FIG. 2: Comparison of the time evolution of the double occupancy and total atom number for different ratios $U / 6 J$. The data was recorded using the $(-9 / 2,-5 / 2)$ spin mixture, with $U / h=3.9 \mathrm{kHz}(3.1 \mathrm{kHz})$ and $J / h=140 \mathrm{~Hz}(200 \mathrm{~Hz})$ for the triangular (round) data points. The lines show fits of the integrated population equations of Eq. 11 The total atom numbers are scaled to the initial values. The inset shows a magnification for short times. Error bars denote the statistical error of at least four identical measurements.

We simultaneously fit the time-dependent populations obtained from Eq. 1 to this dataset and to a corresponding reference dataset without lattice modulation. Since the modulation does not change the losses, this procedure removes the influence of $\tau_{\text {in }}$ and $\tau_{\text {loss }}$, allowing for a reliable determination of the doublon lifetime $\tau_{\mathrm{D}}$. The model and the observation are found to agree very well within experimental uncertainties, as shown in Fig. 2 .

We measure this doublon lifetime for various tunneling and interaction strengths, covering a parameter range 
where $J$ and $U$ each differ by at least a factor of four (inset Fig. 3). The lifetime in units of the tunneling time is plotted logarithmically versus the ratio $U / 6 J$ in Fig. 3 The data is well described by an exponential function:

$$
\frac{\tau_{\mathrm{D}}}{h / J}=C \exp \left(\alpha \frac{U}{6 J}\right) \text {. }
$$

The scaling exponent $\alpha$ is found to be $\alpha=0.82 \pm 0.08$ with $C=1.6 \pm 0.9$. We find fair agreement with our calculations of the doublon lifetime. The systematic deviation of the data for the two spin mixtures [19] seems to indicate that the data show physics beyond Eq. (2).

In the following we argue that this exponential scaling of the doublon lifetime originates from a high order scattering process involving several single atoms as scattering partners. In the preparation of the non-equilibrium state by lattice modulation, we create holes as well as doublons in the bulk and thus drive the system into a compressible state. An isolated doublon has an energy $U$, which it must transfer to other excitations in order to decay. In the compressible state the most relevant excitations are metallic with a typical energy scale of $6 J$. Thus a doublon must scatter with several fermions. The number of scattering partners is on the order of $n=U / 6 \mathrm{~J}$. The matrix element $M$ for the decay rate $\Gamma$ may be estimated via perturbation theory $M \sim \frac{J}{6 J} \times \frac{J}{2 \times 6 J} \times \cdots \times \frac{J}{n \times 6 J}$ and $\Gamma / J \propto M^{2}$. Using Stirling's formula, we then find the same scaling behavior as in Eq. 2. Here $\alpha$ is a parameter

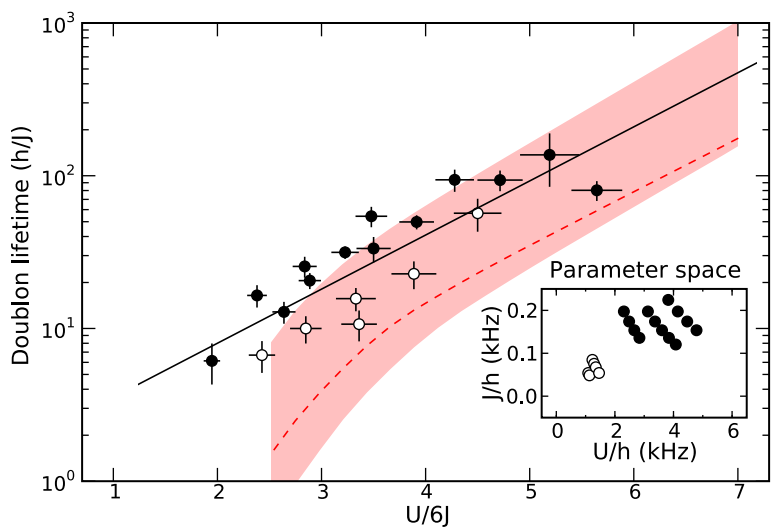

FIG. 3: Semilog plot of doublon lifetime $\tau_{\mathrm{D}}$ vs. $U / 6 J$. The lifetime is extracted from datasets as shown in Fig. 2 Solid and hollow circles denote the $(-9 / 2,-5 / 2)$ and $(-9 / 2,-7 / 2)$ spin mixture respectively, while the dashed line shows the theoretical result at half filling. The solid line is a fit of Eq. 2 to the experimental data, yielding $\alpha=0.82 \pm 0.08$, whereas for the theory curve the asymptotic slope at large $U / 6 J$ is $\alpha_{\mathrm{T}}=0.80$. The shaded corridor was obtained by varying the filling factor in the calculation by \pm 0.3 (which has only a weak effect on the slope). The inset shows the parameters used to realize the different values of $U / 6 J$. Error bars denote the confidence intervals of the lifetime fits and the statistical errors in $U / 6 J$. The systematic errors in $U / 6 J$ and $\tau_{\mathrm{D}} /(h / J)$ are estimated to be $30 \%$ and $25 \%$, respectively. on the order of unity and depends at most logarithmically on $U / 6 J$.

For the quantitative analysis a few assumptions are made: we consider the decay of a single doublon in the background of a homogeneous compressible system. This is justified since most of the doublons are created in the central region of the trap, where the filling is highest, and decay at most within a few sites of where they are produced (the estimated travel distance for a random walk during the decay process is not more than $\sqrt{\tau_{\mathrm{D}} J / h} \lesssim 10$ sites, which is less than the cloud radius). We neglect spin excitations and collisions between doublons, as typical energy transfers in these processes are on the order of $J^{2} / U$, which leads to a subdominant exponential scaling in $U^{2} / J^{2}$. Further, the population of higher bands can be excluded, since $U$ is always smaller than half the band gap. We also note that confinement assisted decay of doublons after quantum tunneling to the edge of the cloud is unlikely, as the accessible confinement energy is smaller than $U$ and the tunneling rate is very small.

The complete Hamiltonian of the system may be written as $H=H_{\mathrm{pf}}+H_{\mathrm{d}}+H_{\mathrm{fd}}$, where $H_{\mathrm{pf}}$ describes the background fermions, $H_{\mathrm{d}}$ is the on-site energy of doublons and $H_{\mathrm{fd}}$ is the interaction of the doublon with the background fermions.

The strong Hubbard repulsion between the fermions leads to the concept of projection, where two fermions are forbidden from occupying the same site. In this case, the fermions can only hop in the presence of a hole on a neighboring site and are governed by the Hamiltonian

$$
H_{\mathrm{pf}}=-J \sum_{\langle i j\rangle, \sigma}\left(1-n_{i, \bar{\sigma}}\right) c_{i, \sigma}^{\dagger} c_{j, \sigma}\left(1-n_{j, \bar{\sigma}}\right),
$$

where $c_{i, \sigma}^{\dagger}\left(c_{i, \sigma}\right)$ is the fermion creation (annihilation) operator and $n_{i, \sigma}$ is the number operator for fermions with spin $\sigma$ ( $\bar{\sigma}$ denotes spin opposite to $\sigma)$. Expanding out this Hamiltonian we obtain $H_{\mathrm{pf}}=H_{\mathrm{f}}+H_{\mathrm{p}}$, with

$$
\begin{aligned}
& H_{\mathrm{f}}=-J \sum_{\langle i j\rangle, \sigma} c_{i, \sigma}^{\dagger} c_{j, \sigma}-\mu \sum_{i, \sigma} c_{i, \sigma}^{\dagger} c_{i, \sigma}, \\
& H_{\mathrm{p}}=J \sum_{\langle i j\rangle, \sigma}\left(n_{i, \bar{\sigma}} c_{i, \sigma}^{\dagger} c_{j, \sigma}+c_{i, \sigma}^{\dagger} c_{j, \sigma} n_{j, \bar{\sigma}}\right),
\end{aligned}
$$

where $H_{\mathrm{f}}$ describes the free Fermi sea and $H_{\mathrm{p}}$ describes the interaction induced by the projection and can be thought of as a process in which a fermion scatters off the Fermi sea and creates a particle-hole pair. We assume that the system is close to half filling (chemical potential $\mu=0$ ), but we checked that the result is not very sensitive to the precise value of the filling as shown by the shaded region in Fig. 3. We neglect the term $n_{i, \bar{\sigma}} c_{i, \sigma}^{\dagger} c_{j, \sigma} n_{j, \bar{\sigma}}$ in $H_{\mathrm{p}}$ as we have checked that it leads to negligibly small corrections to the doublon decay rate 20 .

We now consider the propagation and decay of a doublon in the background state of the projected Fermi sea. 
(a)

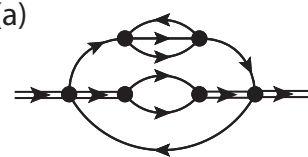

(b)

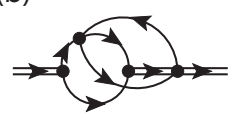

FIG. 4: The double lines represent doublon propagators, and the single lines fermion propagators. (a) Typical doublon propagator diagram showing the creation of particle-hole pairs by both the doublon and the projected fermions as well as annihilation of the doublon into a pair of single fermions. (b) Typical example for a neglected diagram type.

The on-site energy of the doublon is $H_{\mathrm{d}}=U \sum_{i} d_{i}^{\dagger} d_{i}$, where $d^{\dagger}$ is a doublon creation operator. The doublonfermion interaction $H_{\mathrm{fd}}$ is given by

$$
\begin{aligned}
H_{\mathrm{fd}}=J \sum_{\langle i j\rangle}\left(d_{i}^{\dagger} d_{i}\right. & \left.+d_{j}^{\dagger} d_{j}+d_{j}^{\dagger} d_{i}\right) c_{i \sigma}^{\dagger} c_{j \sigma} \\
& +d_{i}\left(c_{i \uparrow}^{\dagger} c_{j \downarrow}^{\dagger}-c_{i \downarrow}^{\dagger} c_{j \uparrow}^{\dagger}\right)+\text { h.c. }
\end{aligned}
$$

where the terms describe: projecting out configurations with a doublon and a fermion on the same site (first and second term), hopping of doublons with back-flow of fermions (third term) and interconversion between a pair of single fermions and a doublon (last term).

We now see that there are two different processes by which the doublon can lose energy. It can create a large number of particle-hole pairs (through the first term in $H_{\mathrm{fd}}$ ), each with an energy of on the order $6 J$, or it can create a high energy particle-hole pair (through $H_{\mathrm{fd}}$ ), which is itself unstable and decays into a shower of particlehole pairs (through the action of $H_{\mathrm{p}}$ ). The last process is the result of strong interaction between the fermions and must be taken into account in order to obtain an accurate estimate of the doublon lifetime.

Our strategy for determining the doublon lifetime is to compute the doublon self-energy $\Sigma(\omega)$ diagrammatically [20] and obtain the decay rate from $\operatorname{Im} \Sigma(U)$, the imaginary part of the self-energy at $\omega=U$. We proceed by first obtaining the Green function for the projected Fermi sea $\left(H_{\mathrm{f}}+H_{\mathrm{p}}\right)$ using a diagrammatic perturbation theory. Next, we use this Green function in a resummation procedure to obtain $\Sigma(\omega)$. These steps can be treated as independent when the doublon density is small, as the presence of the doublons does not change the background fermion Green's functions. Throughout, we follow the principle of maximizing the number of particle-hole pairs (see Fig. 4a) at each order of perturbation theory. We do miss the class of diagrams in which interactions between fermions cannot be described by a fermion self-energy (see Fig. 4b). We carry out our calculations in the zero temperature formalism. However, since we are looking at high energy processes $(\omega \sim U)$, finite temperatures will not have a large effect on the results as long as $T \leq U$ [20].

Our theoretical analysis was constructed to capture the scaling parameter of the doublon lifetime at large $U / 6 \mathrm{~J}$, as it relies on generating a large number of particle-hole pairs. In this regime the theoretically computed value of the scaling exponent is $\alpha_{\mathrm{T}}=0.80$ close to half filling, which agrees well with the experimentally obtained value $\alpha=0.82 \pm 0.08$. For small $U / 6 J$ the theory breaks down, leading to disagreement between experiment and theory in this regime (see Fig. 3). Although the theory is not designed to predict the pre-exponential factor $C$, we find reasonable agreement between theory and experiment. For large $U / 6 J$ losses are expected to mask the observation of very long lifetimes.

In conclusion, we have investigated the nonequilibrium dynamics of fermions in an optical lattice and shown that the lifetime of doublons scales exponentially with the ratio of interaction energy to kinetic energy. We argue that the dominant decay mechanism of doublons is a high order scattering process involving several single particles, and we obtain fair agreement with the experiments based on a perturbation theory calculation. The results have implications also for the simulation of strongly correlated lattice models with ultracold atoms as they pose adiabaticity constraints on the sweep rates for the system parameters. On a qualitative level, the results should also be applicable to bosonic atoms and might help to explain the long equilibration times recently observed in 21].

We thank A. Georges and A. Rosch for insightful discussions, and SNF, NAME-QUAM (EU) and SCALA (EU) for funding. R. S., D. P., and E. D. acknowledge the support of NSF, DARPA, MURI and CUA. E. A. acknowledges support from BSF (ED, EA) and ISF.

* Electronic address: moritz@phys.ethz.ch

[1] U. Schollwöck, Rev. Mod. Phys. 77, 259 (2005).

[2] T. Kinoshita, T. Wenger, D. S. Weiss, Nature (London) 440, 900 (2006).

[3] I. Bloch, J. Dalibard, W. Zwerger, Rev. Mod. Phys. 80, 885 (2008).

[4] M. Köhl, H. Moritz, T. Stöferle, K. Günter, T. Esslinger, Phys. Rev. Lett. 94, 080403 (2005).

[5] R. Jördens, N. Strohmaier, K. Günter, H. Moritz, T. Esslinger, Nature (London) 455, 204 (2008).

[6] U. Schneider et.al., Science 322, 1520-1525 (2008).

[7] V. Perebeinos, P. Avouris, Phys. Rev. Lett. 101, 057401 (2008).

[8] G. Müller et al., Nature Materials 8, 56 (2009).

[9] G. B. Brown, W. Weise, Phys. Rep. 22, 279 (1975).

[10] K. Winkler et al., Nature (London) 441, 853 (2006).

[11] S. D. Huber, A. Rüegg, Phys. Rev. Lett. 102, 065301 (2009).

[12] R. Sensarma et al., Phys. Rev. Lett. 103, 035303 (2009).

[13] C. A. Regal, D. S. Jin, Phys. Rev. Lett. 90, 230404 (2003).

[14] We determine the widths of both resonances by measuring the zero-crossing via dephasing of Bloch oscilla- 
tions 15]. This yields $\Delta B_{-7 / 2,-9 / 2}=7.5 \pm 0.1 \mathrm{G}$ and $\Delta B_{-5 / 2,-9 / 2}=7.6 \pm 0.1 \mathrm{G}$, the latter differing from [13].

[15] M. Gustavsson et al., Phys. Rev. Lett. 100, 080404 (2008)

[16] D. Jaksch, C. Bruder, J. I. Cirac, C. W. Gardiner, P. Zoller, Phys. Rev. Lett. 81, 3108 (1998).

[17] C. Kollath, A. Iucci, I. P. McCulloch, T. Giamarchi, Phys. Rev. A 74, 041604(R) (2006).
[18] F. Hassler, S. D. Huber, Phys. Rev. A 79, 021607(R) (2009).

[19] Separate fits to the two spin mixtures yield values of $\alpha_{(-9 / 2,-5 / 2)}=0.75 \pm 0.10$ and $\alpha_{(-9 / 2,-7 / 2)}=1.00 \pm 0.14$.

[20] R. Sensarma et al., arXiv:1001:3881.

[21] C.-L. Hung, X. Zhang, N. Gemelke, C. Chin, arXiv: $0910.1382(2009)$. 